\title{
HADRONIC REACTIONS IN A DYNAMICAL COUPLED-CHANNEL MODEL*
}

\author{
DEBORAH RÖNCHEN \\ Institut für Kernphysik and Jülich Center for Hadron Physics, \\ Forschungszentrum Jülich, D-52425 Jülich, Germany \\ d.roenchen@fz-juelich.de
}

Published 14 January 2014

\begin{abstract}
The reactions $\pi N \rightarrow \pi N, \eta N, K \Lambda$ and $K \Sigma$ are described in a combined analysis, based on the Jülich dynamical coupled-channel framework. Unitarity, analyticity and other general requirements of the S-matrix are preserved in this Lagrangian-based, field-theoretical approach. The reliable extraction of resonance parameters is possible in terms of poles and residues.
\end{abstract}

Keywords: Baryon spectroscopy; Dynamical coupled channels; Jülich model.

\section{Introduction and Formalism}

To gain insight into the non-perturbative sector of Quantum Chromodynamics the knowledge of the excited hadron spectrum is essential, it provides the connection between experiment and QCD. Most resonances have been identified through elastic $\pi N$ scattering in the past to present day. On the other hand, combining different reactions for resonance extraction allows to determine those states which couple only weakly to $\pi N$. The simultaneous analysis of different final states of pion- and photon-induced reactions is especially interesting regarding the new experimental window that has opened through the recent high-precision photon beam facilities, e.g., at ELSA, JLab and MAMI. Among other approaches, dynamical coupledchannel (DCC) models provide a sophisticated tool to analyze those data on excited baryons as they obey a maximum of theoretical requirements of the $S$-matrix such as analyticity to allow for a reliable extraction of resonances.

The DCC model developed and employed in this study (Jülich model) is based on an approach pursued over the years. ${ }^{1-4}$ Recently, it has been extended to include

\footnotetext{
*This is an Open Access article published by World Scientific Publishing Company. It is distributed under the terms of the Creative Commons Attribution 3.0 (CC-BY) License. Further distribution of this work is permitted, provided the original work is properly cited.
} 
also the states $K \Lambda$ and $K \Sigma .^{5}$ The scattering amplitude is obtained as the solution of a Lippmann-Schwinger equation (Eq. (1)) which guarantees two-body unitarity and approximates three-body unitarity,

$$
\begin{aligned}
& \left\langle L^{\prime} S^{\prime} k^{\prime}\left|T_{\mu \nu}^{I J}\right| L S k\right\rangle=\left\langle L^{\prime} S^{\prime} k^{\prime}\left|V_{\mu \nu}^{I J}\right| L S k\right\rangle \\
& \quad+\sum_{\gamma L^{\prime \prime} S^{\prime \prime}} \int_{0}^{\infty} k^{\prime \prime 2} d k^{\prime \prime}\left\langle L^{\prime} S^{\prime} k^{\prime}\left|V_{\mu \gamma}^{I J}\right| L^{\prime \prime} S^{\prime \prime} k^{\prime \prime}\right\rangle \frac{1}{z-E_{\gamma}\left(k^{\prime \prime}\right)+i \epsilon}\left\langle L^{\prime \prime} S^{\prime \prime} k^{\prime \prime}\left|T_{\gamma \nu}^{I J}\right| L S k\right\rangle .
\end{aligned}
$$

In Eq. (1) $z$ is the scattering energy, $J(L)$ is the total angular (orbital angular) momentum, $S(I)$ is the total spin (isospin), $k\left(k^{\prime}, k^{\prime \prime}\right)$ are the incoming (outgoing, intermediate) momenta, and $\mu, \nu, \gamma$ are channel indices. $E_{\gamma}$ is the on-mass shell energy in channel $\gamma^{3}{ }^{3}$ The pseudo-potential $V$ iterated in Eq. (1) is constructed from an effective interaction based on the Lagrangians of Wess and Zumino, supplemented by additional terms ${ }^{1,2}$ for including the $\Delta$ isobar, the $\omega, \eta, a_{0}$ meson, and the $\sigma$. The channel space is given by $N \pi, N \eta, N \sigma, \Delta \pi, N \rho, \Lambda K$ and $\Sigma K$. The nonresonant interactions are constructed of $t$ - and $u$-channel exchanges of known mesons and baryons, while bare resonances can be considered as $s$-channel processes. The explicit treatment of the background in terms of $t$ - and $u$-channel diagrams introduces strong correlations between the different partial waves and generates a nontrivial energy and angular dependence of the observables. Analyticity is respected in the sense that dispersive, real parts of intermediate states are included, as well as the correct structure of branch points, some of them being in the complex plane, and the off-shell behavior as dictated by the interaction Lagrangians. Thus, a reliable determination of resonance properties given in terms of pole positions and residues is possible.

The extension of the model to photoproduction within a fully gauge-invariant approach has been accomplished recently. ${ }^{6-9}$

In the following, the results of a simultaneous analysis of elastic $\pi N$-scattering and pion-induced $K$ and $\eta$ production within the framework of the Jülich model will be presented. Details on this analysis can be found in Ref. 5 . We perform a resonance analysis of the isospin $I=1 / 2$ and $I=3 / 2$ sector, considering the world data on the set of reactions $\pi^{-} p \rightarrow \eta n, K^{0} \Lambda, K^{0} \Sigma^{0}, K^{+} \Sigma^{-}$, and $\pi^{+} p \rightarrow K^{+} \Sigma^{+}$, together with $\pi N \rightarrow \pi N$ scattering. Within the framework of DCC approaches, this was the first analysis of this type realized until a recent work of the EBAC group, ${ }^{11}$ also presented at this workshop. The approach also includes the three effective $\pi \pi N$ channels $\pi \Delta, \sigma N$ and $\rho N$. The considered energy range has been extended beyond $2 \mathrm{GeV}$ and resonances up to $J=9 / 2$ are included in this study.

The present study is the first step towards a global analysis of pion- and photoninduced production of $\pi N, \eta N, K \Lambda$ and $K \Sigma$. 


\section{Results}

While for the reaction $\pi N \rightarrow \pi N$ the partial waves from the GWU/SAID analysis ${ }^{10}$ are used, for the inelastic channels, $\pi N \rightarrow \eta N$ and $\pi N \rightarrow K Y$, we fit directly to total and differential cross sections as well as to polarization observables. To find out how far our results and, specifically, the extracted resonance parameters are sensitive to the starting conditions of the fitting procedure, we perform two different fits, starting from tow different scenarios in parameter space. Due to the high numerical effort the number of different fits is limited.

Most of the existing data for the inelastic channels was obtained in the 1960's and 70's. Though many experiments have been carried out at different facilities, unfortunately, there are still energy ranges where the data situation is not ideal. All in all we include about 6000 data points in our analysis. The present solution was obtained in a fit procedure using MINUIT on the JUROPA supercomputer at the Forschungszentrum Jülich.

In the previous analysis, ${ }^{4}$ the reaction $\pi^{+} p \rightarrow K^{+} \Sigma^{+}$and $\pi N$ scattering were considered and only resonance parameters were fitted. In this study, in addition the important $T^{\mathrm{NP}}$ parameters are varied. Those are the cut-offs of the form factors in $t$ - and $u$-channel exchange diagrams.

One bare $s$-channel state is included in each of the $I=1 / 2$ partial waves, except for the $S 11$ and $P 11$ where we have two. In the $I=3 / 2$ sector, one bare $s$-channel state is included in each partial waves and two are included in P33. These states couple to all channels $\pi N, \rho N, \eta N, \pi \Delta, K \Lambda$ and $K \Sigma$ if allowed by isospin. In total, we have 196 free parameters, of which 128 are resonance parameters (bare masses and couplings) and 68 belong to the $T^{\mathrm{NP}}$ part ( $t$ - and $u$-channel exchanges). The values of the parameters are quoted in Ref. 5. In Figs. 1 and 2 selected fit results are shown. The full result and the extracted pole positions and residues of the resonances can be found in Ref. 5 .
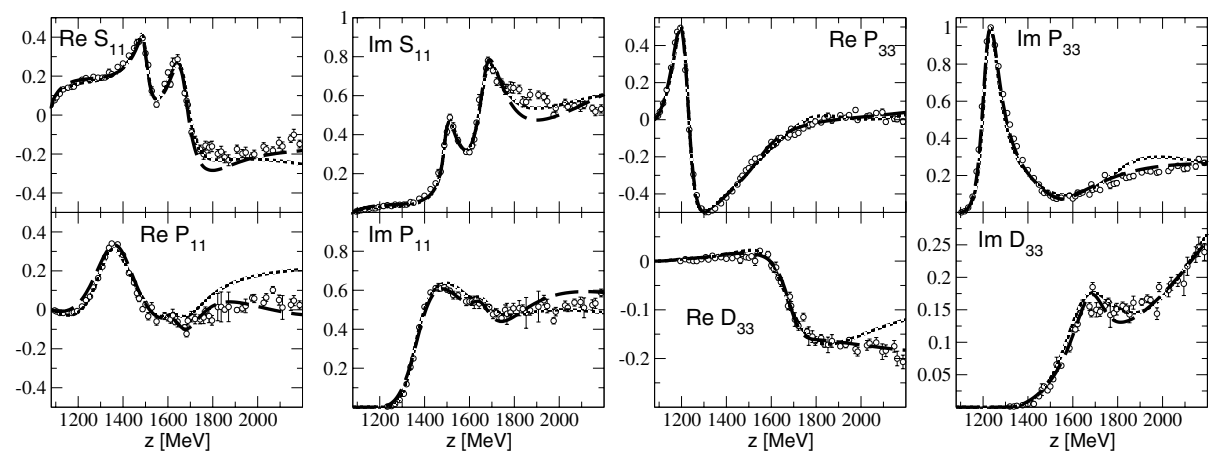

Fig. 1. Reaction $\pi N \rightarrow \pi N$, real and imaginary part of the $S 11, P 11, P 33$ and $D 33$ partial waves. Solid lines: fit A. Dashed lines: fit B. Data points: GWU/SAID partial wave analysis (single energy solution) from Ref. 10. Color in Ref. 5. 

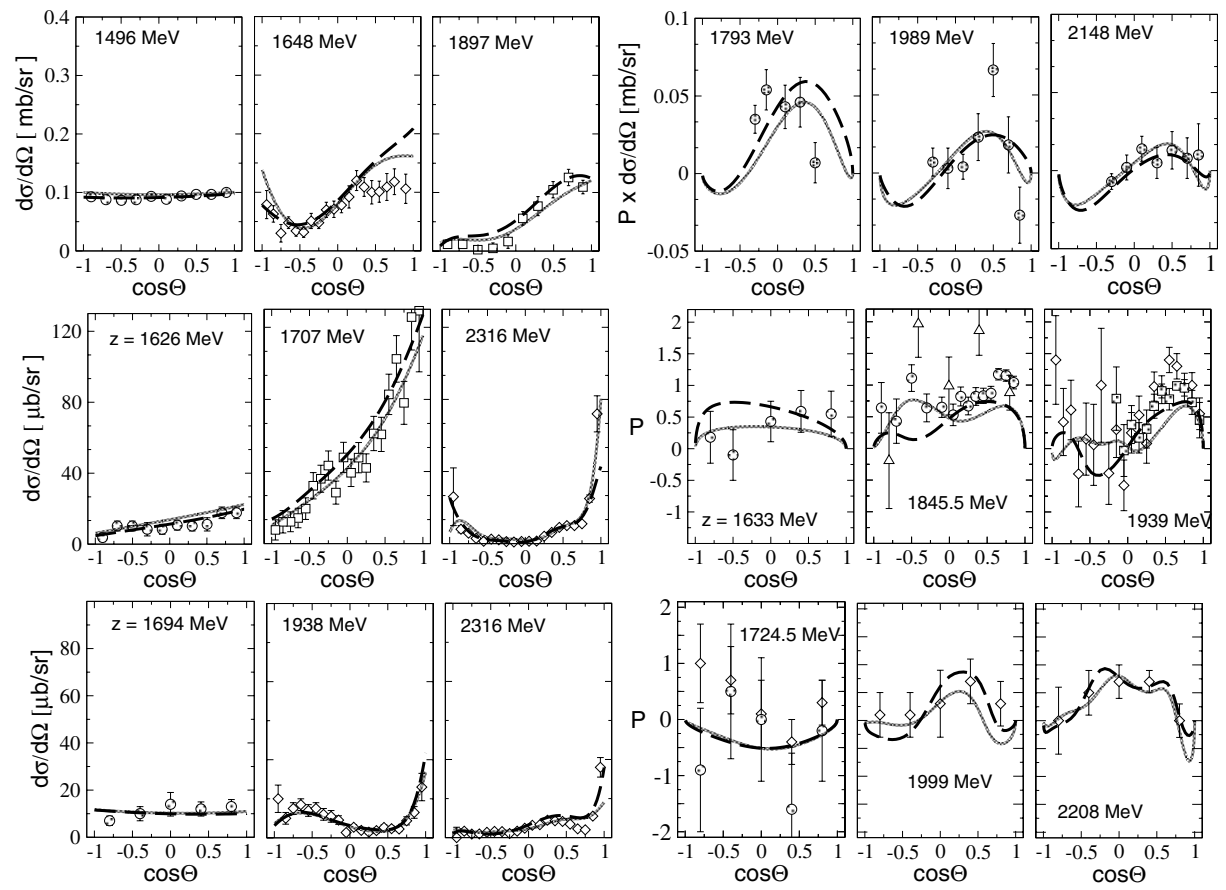

Fig. 2. Differential cross section (left) and polarization (right) for the reactions $\pi^{-} p \rightarrow \eta n$ (upper row), $\pi^{-} p \rightarrow K^{0} \Lambda$ (middle) and $\pi^{-} p \rightarrow K^{0} \Sigma^{0}$ (lower). Solid lines: fit A. Dashed lines: fit B. Color and data references in Ref. 5 .

\section{Acknowledgement}

This work has been carried out in collaboration with M. Döring, F. Huang, H. Haberzettl, J. Haidenbauer, C. Hanhart, S. Krewald, U.-G. Meißner and K. Nakayama. I am also grateful to the German Academic Exchange Service (DAAD) for financial support within a "DAAD-Doktorandenstipendium".

\section{References}

1. O. Krehl, C. Hanhart, S. Krewald and J. Speth, Phys. Rev. C 62, 025207 (2000).

2. A. M. Gasparyan, J. Haidenbauer, C. Hanhart and J. Speth, Phys. Rev. C 68, 045207 (2003).

3. M. Döring, C. Hanhart, F. Huang, S. Krewald and U.-G. Meißner, Nucl. Phys. A 829, 170 (2009).

4. M. Döring, C. Hanhart, F. Huang, S. Krewald, U.-G. Meißner and D. Rönchen, Nucl. Phys. A 851, 58 (2011).

5. D. Rönchen, M. Döring, F. Huang, H. Haberzettl, J. Haidenbauer, C. Hanhart, S. Krewald and U. -G. Meißner et al., Eur. Phys. J. A 49, 44 (2013)

6. H. Haberzettl, Phys. Rev. C 56 (1997) 2041.

7. H. Haberzettl, K. Nakayama, S. Krewald, Phys. Rev. C74 (2006) 045202.

8. H. Haberzettl, F. Huang and K. Nakayama, Phys. Rev. C 83 (2011) 065502. 
9. F. Huang, M. Döring, H. Haberzettl, J. Haidenbauer, C. Hanhart, S. Krewald, U.-G. Meißner and K. Nakayama, Phys. Rev. C 85, 054003 (2012).

10. R. A. Arndt, W. J. Briscoe, I. I. Strakovsky and R. L. Workman, Phys. Rev. C 74, 045205 (2006).

11. H. Kamano, S. X. Nakamura, T. -S. H. Lee and T. Sato, arXiv:1305.4351 [nucl-th]. 